\title{
Determination of Time-Dependent Coefficients in Time-Fractional Diffusion Equations by Variational Iteration Method
}

\author{
Mimi Li ${ }^{1}$, Gongsheng $\mathrm{Li}^{1}$, Zhiyuan $\mathrm{Li}^{1}$ \& Xianzheng $\mathrm{Jia}^{1}$ \\ ${ }^{1}$ School of Mathematics and Statistics, Shandong University of Technology, Zibo, China \\ Correspondence: Gongsheng Li, School of Mathematics and Statistics, Shandong University of Technology, Zibo, China. \\ E-mail: ligs@sdut.edu.cn
}

Received: December 2, 2019 Accepted: January 13, 2020 Online Published: January 31, 2020

doi:10.5539/jmr.v12n1p74

URL: https://doi.org/10.5539/jmr.v12n1p74

\begin{abstract}
Inverse problems of determining time-dependent coefficients in partial differential equations are difficult to deal with in general cases. The variational iteration method is introduced to determine the time-dependent coefficient in the fractional diffusion equation as well as the solution of the forward problem. By utilizing the additional condition and the property of the fractional derivative, an expression of the unknown is derived by which a nonlinear dynamical differential equation is obtained. The variational iteration method is applied to solve the nonlinear system and the time-dependent coefficient can be reconstructed in a semi-analytical form. Such method can give explicit expression of the solution in the meaning of approximation, or exact solution to the inverse problem in some cases. Several examples are presented to demonstrate feasibility and effectiveness of the proposed method for inverse time-dependent coefficient problems in the fractional diffusion equations.
\end{abstract}

Keywords: time fractional diffusion equation, inverse problem, time-dependent coefficient, variational iteration method, series of approximate solutions

\section{Introduction}

The fractional differential equations and their applications have attracted much attention during the last two decades. If the usual integer-order derivative in a classical diffusion equation is replaced with a fractional derivative of any real number in time or space, a time or space fractional diffusion equation is established, which has more and more applications in the fields of physics, mechanics, environmental science and hydrology, etc. On concrete applications of mathematical models and simulation, inverse problems are always encountered with in which we need to identity and determine some unknowns in the model using suitable additional information. As for inverse problems arising in the time/space fractional diffusion equations, we refer to Chen, Liu, Jiang, Turner and Burrage (2016), Cheng, Nakagawa, Yamamoto and Yamazaki (2009), Chi, Li and Jia (2011), Jin and Rundell (2015), Li, Zhang, Jia and Yamamoto (2013), Li, Imanuvilov and Yamamoto(2016), Liu and Yamamoto(2010), Liu, Rundell and Yamamoto (2016), Luchko, Rundell and Yamamoto(2013), Miller and Yamamoto (2013), Sakamoto and Yamamoto (2011), Wei, L. Li and S. Li (2016), Yamamoto and Zhang (2012), and recently see Fan, Liu and Jiang (2018), Kian, Oksanen, Soccorsi and Yamamoto (2018), Ruan, Zhang and Wang (2018), Sun, Li and Jia (2017), Sun, Yan and Wei (2019) and the references therein. It is noted that almost inverse problems in the fractional diffusion equations are concerned with the fractional orders, the source term, or the space-dependent coefficient, it is still difficult for inverse problems of determining time-dependent coefficients in partial differential equations in general cases, especially for fractional diffusion models with time-dependent coefficients.

On the other hand, the inversion methods for solving inverse problems are often proposed based on the minimization for the cost functional and regularization strategies. Such kind of algorithms involve in gradient-type iterations and computations of the forward problem repeatedly, and the computational cost is expansive. From viewpoint of engineering application it is important and convenient to get an approximate solution with an explicit expansion for a mathematical model. The main motivation of this work is to drive an inversion method which is independent upon the gradient computation and can give a fast and accurate approximation for the solution of an inverse time-dependent coefficient problem.

As we know the variational iteration method is a semi-analytical method to get a solution of a linear/nonlinear algebra/differential equation arising from mathematical physics and engineering science, see the original work by He (1997, 2000, 2007), etc. Such variational iteration method can provide us with a possibility to analyze some nonlinear models because of its no specific requirements for the nonlinear operators, see Chang (2016), Nadeem, Li and Ahmad (2019), Tapaswini, Chakraverty and Behera (2015), Zhao, Zhu, Yu and Xu (2010) for example, and it was employed to find the exact solution or approximate solutions of fractional differential equations with effectiveness, see Biazar, Gholamin and 
Hosseini (2010), Dehghan and Tatari (2006), Inc (2008), S. Kumar, A. Kumar and Argyros (2017), Wu (2012), Yulita (2009), etc.

By the variational iteration method, we consider the inverse problem of determining the time-dependent diffusion coefficient $a(t)$ in the time-fractional diffusion equation

$$
\partial_{t}^{\alpha} u-a(t) \Delta u=f(x, t),
$$

and the inverse problem of determining the average flow velocity $v(t)$ in the mobile/immobile time-fractional diffusion equation

$$
u_{t}+r \partial_{t}^{\alpha} u-a(t) \Delta u+v(t) \nabla u=f(x, t),
$$

respectively, where $\partial_{t}^{\alpha} u$ denotes the $\alpha$-order Caputo's fractional derivative of $u=u(x, t)$ at $t>0$ for $0<\alpha<1$, see the definition of fractional calculus by Kilbas, Srivastara and Trujillo (2006), Podlubny (1999).

For the inverse problem of determining the diffusion coefficient $a(t)$ in Eq.(1.1) using the boundary measurement, a uniqueness and a fixed point iteration algorithm are presented by Zhang (2016). As for Eq.(1.2), there are a few of researches on numerical methods for the forward problem, see Jiang and Liu (2017), Liu, Meerschaert, McGough, Zhuang and Liu (2013), Q. Liu, F. Liu, Turner, Anh and Gu (2014), Zhang, Liu, Phanikumar and Meerschaert (2013) for example, however, the related inverse problems seem to be paid less attention in the literatures.

This paper will give numerical determination for the above inverse problems of determining time-dependent coefficients using the variational iteration method. Although the variational iteration method has been utilized to solve nonlinear problems not only for classical integer-order diffusion equations but also for fractional diffusion equations, there are few researches on inverse coefficient problems by the method. One purpose of this work is try to apply the variational iteration method to solve some inverse coefficient problems in the fractional diffusion equations, especially for determination of a time-dependent coefficient which is difficult to cope with in general cases. We will perform the approximate inversions for the considered inverse time-dependent problems using the variational iteration method, and the exact solution can be obtained in some cases. The rest of the paper is organized as follows.

In Section 2, the variational iteration method for solving a forward problem and an inverse coefficient problem in a differential equation is introduced. In Section 3 by using the properties of fractional calculus, the variational iteration method is applied to solve the inverse problem of determining a time-dependent diffusion coefficient in the time-fractional diffusion equation based on the additional data at one interior-point, and in Section 4 a time-dependent flow velocity in the mobile/immobile time fractional advection-dispersion equation is recovered also by the variational iteration method. Finally concluding remarks are given in Section 5.

\section{The Variational Iteration Method}

\subsection{The Variational Iteration Method for Forward Problem}

Consider a second-order partial differential equation in an abstract form

$$
F\left(u, u_{t}, u_{t t}, u_{x}, u_{x x}\right)=0,
$$

with specified initial boundary conditions. The variational iteration method changes the equation to a correction functional in $t$-direction in the form

$$
u_{n+1}(x, t)=u_{n}(x, t)+\int_{0}^{t} \lambda(t, s) F\left(u_{n}, u_{n s}, u_{n s s}, \tilde{u}_{n x}, \tilde{u}_{n x x}\right) d s,
$$

where $\lambda$ is a general Lagrange multiplier which is identified optimally by variational theory, $u_{n}$ is the $\mathrm{n}$-th approximate solution, and $\tilde{u}_{n}$ denotes a restricted variation, i.e., $\delta \tilde{u}_{n}=0$. Let the variation $\delta u_{n+1}=0$ and an optimal value of $\lambda$ is solved, and then an iteration formula for solving the equation (2.1) is constructed by which an approximate analytical solution can be obtained.

Rewrite (2.2) with

$$
u_{n+1}(x, t)-u_{n}(x, t)=\int_{0}^{t} \lambda(t, s) F\left(u_{n}, u_{n s}, u_{n s s}, u_{n x}, u_{n x x}\right) d s .
$$

Roughly speaking, the optimal value of $\lambda$ must be chosen to minimize the residual such that the limitation of $u_{n}$ exists as $n \rightarrow \infty$. On the other hand, suppose that the function $F$ is continuous and the forward problem has unique solution. Then $u^{*}=\lim _{n \rightarrow \infty} u_{n}$ is just the solution satisfying $F\left(u^{*}, u_{t}^{*}, u_{t t}^{*}, u_{x}^{*}, u_{x x}^{*}\right)=0$.

Actually, by (2.2) define a mapping

$$
A[u]=u(x, t)+\int_{0}^{t} \lambda(t, s) F\left(u, u_{s}, u_{s s}, u_{x}, u_{x x}\right) d s .
$$


A sufficient condition for the convergence is the operator $A$ is a contraction, and its fixed point is also the solution of the equation (2.2).

\subsection{The Variational Iteration Method for Inverse Coefficient Problem}

Consider a general differential equation involving an unknown coefficient function $p=p(t)$ given as

$$
F\left(u, u_{t}, u_{x}, u_{x x}, p\right)=f(x, t), x \in \Omega, 0<t<T,
$$

with specified initial boundary conditions. Suppose that we have some additional information given at one point $x^{*} \in \bar{\Omega}$

$$
u\left(x^{*}, t\right)=E(t), x^{*} \in \bar{\Omega},
$$

We are to determine the time-dependent coefficient $p=p(t)$ in (2.5) with the overposed condition (2.6) using the variational iteration method.

Firstly we have to deduce an expression of the coefficient $p=p(t)$ by the forward problem and the additional condition, denoted by

$$
p(t)=G\left(u, u_{t}, u_{x}, u_{x x}, f, E\right),
$$

which is a key step for the application of the variational iteration method in the inverse coefficient problem.

Next, substituting (2.7) into the equation (2.5), there has

$$
F\left(u, u_{t}, u_{x}, u_{x x}, G\left(u, u_{t}, u_{x}, u_{x x}, f, E\right)\right)=0 .
$$

which is always a nonlinear equation on $u=u(x, t)$.

Utilizing the variational iteration method to the equation (2.8) with suitable initial iteration, an approximate solution, or the exact solution of $u=u(x, t)$ in some cases can be obtained, and the coefficient $p(t)$ is determined by (2.7).

\section{Inversion for Diffusion Coefficient in Time-FDE}

Consider the time fractional diffusion equation with time-dependent diffusion coefficient

$$
\partial_{t}^{\alpha} u-a(t) u_{x x}=f(x, t), 0<x<1,0<t<T,
$$

with specified initial boundary conditions, where $\partial_{t}^{\alpha}$ is Caputo's derivative for $\alpha \in(0,1)$.

The inversion problem is determine the diffusion coefficient $a=a(t)$ given the interior measurement at a fixed point $x^{*} \in(0,1)$.

$$
u\left(x^{*}, t\right)=E(t), 0<t \leq T .
$$

\subsection{Transformation of the Inverse Coefficient Problem}

For the aim of utilizing the variational iteration method to solve the inverse coefficient problem we need some preliminary knowledge of the fractional calculus, see Kilbas, Srivastara and Trujillo(2006), Podlubny(1999) for example.

The $\alpha$-order Riemann-Liouville integral operator $I^{\alpha}$, defined by

$$
I^{\alpha} f(t)=\frac{1}{\Gamma(\alpha)} \int_{0}^{t} \frac{f(\tau)}{(t-\tau)^{1-\alpha}} d \tau .
$$

The $\alpha$-order $(0<\alpha<1)$ Riemann-Liouville derivative, ${ }^{R L} D_{t}^{\alpha} f$, defined by

$$
{ }^{R} D_{t}^{\alpha} f(t)=\frac{d}{d t}\left(I^{1-\alpha} f(t)\right) .
$$

The $\alpha$-order $(0<\alpha<1)$ Caputo's derivative, $\partial_{t}^{\alpha} f$, defined by

$$
\partial_{t}^{\alpha} f(t)=I^{1-\alpha}\left(\frac{d f}{d t}\right) .
$$

There hold for $0<\alpha<1$

$$
\partial_{t}^{\alpha} I^{\alpha} f(t)=f(t)
$$

and

$$
I^{\alpha} \partial_{t}^{\alpha} f(t)=f(t)-f(0)
$$


Now consider the equation (3.1) again. Since

$$
I^{\alpha} \partial_{t}^{\alpha} u(x, t)=u(x, t)-u(x, 0),
$$

By using the additional information we have

$$
E(t)-u\left(x^{*}, 0\right)=I^{\alpha}\left[a(t) u_{x x}\left(x^{*}, t\right)+f\left(x^{*}, t\right)\right] .
$$

Then it holds

$$
\begin{aligned}
\partial_{t}^{\alpha} E(t) & =\partial_{t}^{\alpha}\left[E(t)-u\left(x^{*}, 0\right)\right] \\
& =\partial_{t}^{\alpha}\left\{I^{\alpha}\left[a(t) u_{x x}\left(x^{*}, t\right)+f\left(x^{*}, t\right)\right]\right\} \\
& =a(t) u_{x x}\left(x^{*}, t\right)+f\left(x^{*}, t\right) .
\end{aligned}
$$

Suppose that $u_{x x}\left(x^{*}, t\right) \neq 0$, we get

$$
a(t)=\frac{\partial_{t}^{\alpha} E(t)-f\left(x^{*}, t\right)}{u_{x x}\left(x^{*}, t\right)} .
$$

On the other hand, by taking $1-\alpha$-order Riemann-Liouville differential operator on equation (3.1) there holds

$$
{ }^{R} D_{t}^{1-\alpha} \partial_{t}^{\alpha} u(x, t)={ }^{R} D_{t}^{1-\alpha}\left[a(t) u_{x x}+f(x, t)\right] .
$$

Noting that ${ }^{R} D_{t}^{1-\alpha}=\frac{\partial}{\partial t} I^{\alpha}$, we have

$$
\frac{\partial u}{\partial t}={ }^{R L} D_{t}^{1-\alpha}\left[a(t) u_{x x}+f(x, t)\right] .
$$

Substituting (3.9) into (3.11) we have

$$
\frac{\partial u}{\partial t}={ }^{R L} D_{t}^{1-\alpha}\left[\frac{\partial_{t}^{\alpha} E(t)-f\left(x^{*}, t\right)}{u_{x x}\left(x^{*}, t\right)} u_{x x}(x, t)+f(x, t)\right] .
$$

\subsection{Inversion for the Diffusion Coefficient}

Now we are ready to employ the variational iteration method to solve the inverse coefficient problem. By the VIM, define a corrected functional along $t$-direction given as

$$
\begin{aligned}
u_{n+1}(x, t)= & u_{n}(x, t)+\int_{0}^{t} \lambda(\tau)\left\{\frac{\partial u_{n}}{\partial \tau}(x, \tau)\right. \\
& \left.-{ }^{R} D_{\tau}^{1-\alpha}\left[\frac{\partial_{\tau}^{d} E(\tau)-f\left(x^{*}, \tau\right)}{u_{n x x}\left(x^{*}, \tau\right)} \tilde{u}_{n x x}(x, \tau)+f(x, \tau)\right]\right\} d \tau,
\end{aligned}
$$

where $\lambda(\tau)$ is the general Lagrange multiplier, and $\tilde{u}_{n}$ is a confined variational which satisfies $\delta \tilde{u}_{n}=0$ as discussed by He (2007).

Noting that $\delta \tilde{u}_{n}=0$, we take the first-variation on $u_{n}$ for (3.13), and let $\delta u_{n+1}=0$, there holds

$$
\delta u_{n+1}(x, t)=\delta u_{n}(x, t)+\int_{0}^{t} \lambda(\tau) \frac{\partial\left(\delta u_{n}\right)}{\partial \tau} d \tau=0 .
$$

By integration by parts, and thanks to $\delta u_{n}(x, 0)=0$ there must have

$$
\left\{\begin{array}{l}
\lambda^{\prime}(\tau)=0 \\
1+\lambda(t)=0
\end{array}\right.
$$

from which we get $\lambda(\tau)=-1$ for $\tau \in[0, t]$. Thus by (3.13) we have the iteration formula

$$
\begin{aligned}
u_{n+1}(x, t)= & u_{n}(x, t)-\int_{0}^{t}\left\{\frac{\partial u_{n}}{\partial \tau}(x, \tau)\right. \\
& \left.-{ }^{R} D_{\tau}^{1-\alpha}\left[\frac{\partial_{\tau}^{*} E(\tau)-f\left(x^{*}, \tau\right)}{u_{n x x}\left(x^{*}, \tau\right)} u_{n x x}(x, \tau)+f(x, \tau)\right]\right\} d \tau,
\end{aligned}
$$

for $n=0,1, \cdots$. With the initial value function $u_{0}(x)$ as the initial iteration, we can get a series of $u_{n}(x, t)$ by (3.15) which are the approximate solutions of the forward problem, and then the diffusion coefficient $a(t)$ is computed by (3.9).

\subsubsection{Example 3.1}

Let the exact solution of the equation (3.1) be $u(x, t)=x^{3}+t^{2}$, and the diffusion coefficient be $a(t)=1+t$, and the source be

$$
f(x, t)=\frac{2 t^{2-\alpha}}{\Gamma(3-\alpha)}-6 x(1+t) .
$$


Suppose that the additional condition is given at $x=\frac{1}{2}$ :

$$
E(t)=u\left(\frac{1}{2}, t\right)=\frac{1}{8}+t^{2}
$$

By the variational iteration formula (3.15), we have

$$
\begin{aligned}
u_{n+1} & =u_{n}-\int_{0}^{t}\left\{\frac{\partial u_{n}}{\partial \tau}\right. \\
& \left.-{ }^{R} D_{\tau}^{1-\alpha}\left[\frac{\partial \tau}{\tau_{\tau} E(\tau)-f\left(x^{*}, \tau\right)} u_{x x}+f(x, \tau)\right]\right\} d \tau \\
& =u_{0}(x)+\int_{0}^{t} R D_{\tau}^{1-\alpha}\left[\frac{x_{\tau}^{*} E(\tau)-f\left(x^{*}, \tau\right)}{u_{x x}\left(x^{*}, \tau\right)} u_{x x}+f(x, \tau)\right] d \tau
\end{aligned}
$$

Choosing the initial iteration $u_{0}=u(x, 0)=x^{3}$, we get by computation

$$
\begin{aligned}
u_{1} & =u_{0}+\int_{0}^{t} R D_{\tau}^{1-\alpha}\left[\frac{2}{\Gamma(3-\alpha)} \tau^{2-\alpha}\right] d \tau \\
& =u_{0}+\int_{0}^{t} R D_{\tau}^{1-\alpha} \partial_{\tau}^{\alpha}\left(\tau^{2}\right) d \tau \\
& =x^{3}+t^{2}
\end{aligned}
$$

which is the exact solution of the forward problem. Then the inversion coefficient is worked out by (3.9) given as

$$
\begin{aligned}
a(t) & =\frac{\partial_{t}^{\alpha} E(t)-f\left(\frac{1}{2}, t\right)}{u_{x \alpha}\left(\frac{1}{2}, t\right)} \\
& \left.=\frac{2 t^{2}-\alpha}{\Gamma(3-\alpha)}-\frac{2 t^{-\alpha}-\alpha}{\Gamma(3-\alpha)}-3(t+1)\right] \\
& =t+1,
\end{aligned}
$$

which is just the exact the diffusion coefficient.

\subsubsection{Example 3.2}

Let the exact solution be $u(x, t)=\exp (-t)\left(x^{2}+1\right)$, and $a(t)=\exp (t)$, and the additional condition also given at $x=\frac{1}{2}$

$$
E(t)=u\left(\frac{1}{2}, t\right)=\frac{5}{4} \exp (-t)
$$

By the variational iteration method as utilized in the above, we have by setting $u_{0}=u(x, 0)=x^{2}+1$

$$
\begin{aligned}
u_{1} & =u_{0}+\int_{0}^{t} R D_{\tau}^{1-\alpha}\left[\partial_{\tau}^{\alpha} E(\tau)-f\left(\frac{1}{2}, \tau\right)+f(x, \tau)\right] d \tau \\
& =u_{0}+\int_{0}^{t} R D_{\tau}^{1-\alpha} \partial_{\tau}^{\alpha}\left[E(\tau)+\left(x^{2}+1\right) e^{-\tau}-\frac{5}{4} e^{-\tau}\right] d \tau \\
& =\left(x^{2}+1\right) \exp (-t),
\end{aligned}
$$

which is the exact solution of the forward problem, and the inversion diffusion coefficient is computed also by (3.9)

$$
\begin{aligned}
a(t) & =\frac{\partial_{t}^{\alpha} E(t)-f\left(\frac{1}{2}, t\right)}{u_{x x}\left(\frac{1}{2}, t\right)} \\
& =\frac{\left.\frac{2 t^{2}-x}{\Gamma(3-\alpha)}-\frac{22^{2}-\alpha}{\Gamma(3-\alpha)}-2\right]}{2 \exp (-t)} \\
& =\exp (t) .
\end{aligned}
$$

\section{Inversion for Average Velocity in Mobile/Immobile Fractional Transport Model}

Consider the time fractional diffusion equation in a bounded domain $(0, l)$ for $l>0$

$$
u_{t}+r \partial_{t}^{\alpha} u-a(t) u_{x x}+v(t) u_{x}=f(x, t), 0<x<l, 0<t<T,
$$

with the initial value condition:

$$
u(x, 0)=u_{0}(x), 0 \leq x \leq l,
$$

and the specified Neumann boundary conditions.

Suppose that the average flow velocity $v(t)$ in the model is unknown, we are to determine it using the out-flow measurements at $x=l$ :

$$
u(l, t)=E(t), 0<t \leq T .
$$

In addition, in order to utilizing the variational iteration method to solve the inverse coefficient problem, we need the conditions that the right-boundary function $u_{x}(l, t) \neq 0$ for $t \in[0, T]$, and the coefficient $r>0$ in (4.1). 
By the equation (4.1) we have

$$
\partial_{t}^{\alpha} u=\frac{1}{r}\left[f(x, t)-u_{t}+a(t) u_{x x}-v(t) u_{x}\right] .
$$

Similarly as done in the subsection 3.1, noting the condition $u_{x}(l, t) \neq 0$, by utilizing the properties of the fractional calculus and the additional condition, we get an expression of the average flow velocity given by

$$
v(t)=\frac{f(l, t)-E^{\prime}(t)+a(t) u_{x x}(l, t)-r \partial_{t}^{\alpha} E(t)}{u_{x}(l, t)} .
$$

Substituting (4.5) into equation (4.1), we have

$$
\begin{aligned}
& u_{t}(x, t)+r \partial_{t}^{\alpha} u(x, t)-a(t) u_{x x}(x, t) \\
+\quad & \frac{f(l, t)-E^{\prime}(t)+a(t) u_{x}(l, t)-r \partial_{t}^{\alpha} E(t)}{u_{x}(l, t)} u_{x}(x, t)-f(x, t) \\
= & 0 .
\end{aligned}
$$

Now we are ready to employ the variational iteration method to solve the inverse coefficient problem. By utilizing the VIM to the equation (4.6), there is

$$
\begin{aligned}
u_{n+1}(x, t) & =u_{n}(x, t)-\int_{0}^{t}\left[u_{n \tau}(x, \tau)+r \partial_{\tau}^{\alpha} u(x, \tau)-a(\tau) u_{n x x}(x, \tau)\right. \\
& \left.+\frac{f(l, \tau)-E^{\prime}(\tau)+a(\tau) u_{n x x}(l, \tau)-r \partial_{\tau}^{\alpha} E(\tau)}{u_{n x}(l, \tau)} u_{n x}(x, \tau)-f(x, \tau)\right] d \tau,
\end{aligned}
$$

for $n=0,1, \cdots$. With the initial value function $u_{0}(x)$ as the initial iteration, we can get a series of $u_{n}(x, t)$ by $(4.7)$ which are the approximate solutions of the forward problem, and then the average flow velocity $v(t)$ is computed by (4.5).

\subsection{Example 4.1}

Let $l=1$ and $T=1$, and the exact solution of the forward problem be

$$
u(x, t)=\exp (-x)\left(t^{2}+1\right)
$$

and the exact flow velocity be

$$
v(t)=\frac{1}{1+t^{2}} .
$$

In addition, the time-scale coefficient $r=\frac{1}{2}$, the diffusion coefficient $a(t)=1+\frac{t}{1+t^{2}}$, and the additional information $E(t)=\exp (-1)\left(t^{2}+1\right)$, and the source term

$$
f(x, t)=\exp (-x)\left(\frac{t^{2-\alpha}}{\Gamma(3-\alpha)}-t^{2}+t-2\right) .
$$

We are to reconstruct the solution $u(x, t)$ and the flow velocity $v(t)$ by the variational iteration method.

Noting that

$$
E^{\prime}(t)=2 t \exp (-1), \partial_{t}^{\alpha} E(t)=\exp (-1) \frac{2 t^{2-\alpha}}{\Gamma(3-\alpha)},
$$

we have by the variational iteration formula (4.7) by choosing $u_{0}=\exp (-x)$

$$
\begin{aligned}
u_{1} & =u_{0}-\int_{0}^{t}\left[-a(\tau) \exp (-x)+\frac{f(1, \tau)-E^{\prime}(\tau)+a(\tau) \exp (-1)-r \partial_{\tau}^{\alpha} E(\tau)}{\exp (-1)} \exp (-x)-f(x, \tau)\right] d \tau \\
& =u_{0}+\int_{0}^{t}\left[\left(\tau^{2}+\tau+2\right) \exp (-x)+f(x, \tau)\right] d \tau \\
& =\exp (-x)\left[\left(t^{2}+1\right)+\frac{t^{3-\alpha}}{\Gamma(4-\alpha)}\right] .
\end{aligned}
$$

Consequently by the iteration process follows that

$$
\begin{aligned}
& u_{2}=\exp (-x)\left[\left(t^{2}+1\right)-\frac{t^{4-2 \alpha}}{2 \Gamma(5-2 \alpha)}\right] \\
& u_{3}=\exp (-x)\left[\left(t^{2}+1\right)+\frac{t^{5-3 \alpha}}{2^{2} \Gamma(6-3 \alpha)}\right]
\end{aligned}
$$

and

$$
u_{n}=\exp (-x)\left[\left(t^{2}+1\right)+(-1)^{n-1} \frac{t^{n(1-\alpha)+2}}{2^{n-1} \Gamma(n(1-\alpha)+3)}\right] .
$$




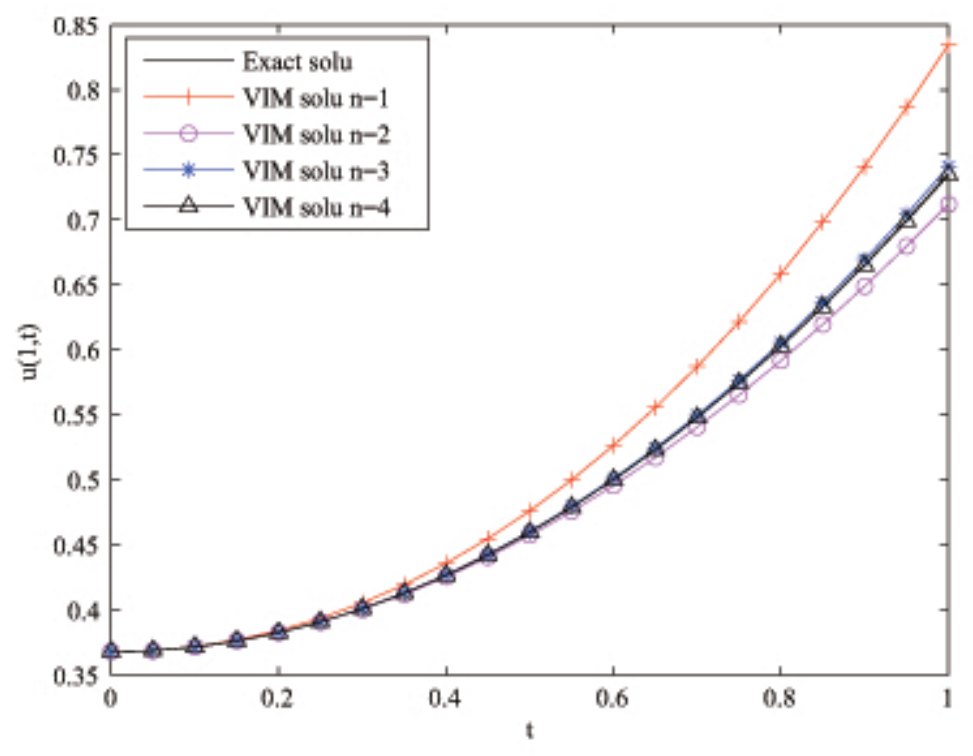

Figure 1. Exact solution and approximate solutions by VIM in Ex.4.1

For any given $\alpha \in(0,1)$ and $T<\infty$, there holds

$$
\lim _{n \rightarrow \infty} \frac{T^{n \alpha}}{2^{n} \Gamma(n \alpha)}=0
$$

Therefore we get

$$
u(x, t)=\lim _{n \rightarrow \infty} u_{n}(x, t)=\exp (-x)\left(t^{2}+1\right)
$$

which is the exact solution of the forward problem.

Furthermore, we have by (4.5)

$$
\begin{aligned}
v_{n}(t) & =\frac{f(1, t)-E^{\prime}(t)+a(t) \frac{\partial^{2} u_{n}}{\partial x^{2}}(1, t)-r \partial_{t}^{\alpha} E(t)}{\frac{\partial u_{n}}{\partial x}(1, t)} \\
& =\frac{f(1, t)-2 t \exp (-1)+a(t) u_{n}(1, t)-\frac{t^{2-\alpha}}{\Gamma(3-\alpha)} \exp (-1)}{-u_{n}(1, t)} .
\end{aligned}
$$

Utilizing (4.12), (4.10) and noting $\frac{t^{2}+t+1}{t^{2}+1} \leq \frac{3}{2}$ and (4.13), we finally get

$$
v_{n}(t)=\frac{1+\frac{t^{2}+t+1}{t^{2}+1} \frac{(-1)^{n} t^{n(1-\alpha)+2}}{2^{n-1} \Gamma(n(1-\alpha)+3)}}{t^{2}+1+\frac{(-1)^{n-1} t^{n(1-\alpha)+2}}{2^{n-1} \Gamma(n(1-\alpha)+3)}} \rightarrow \frac{1}{t^{2}+1}(n \rightarrow \infty),
$$

which is just the exact flow velocity. Moreover, the approximate solutions of $u_{n}(x, t)(n=1,2,3,4)$ by the variational iteration method, and the exact solution $u(x, t)$ for $\alpha=0.4$ and $x=1$ are plotted in Fig. 1, respectively, where VIM denotes the variational iteration method.

\subsection{Example 4.2}

Also let $l=1$ and $T=1$, and the exact solution in this example be

$$
u(x, t)=x^{2}+(t+1)^{2},
$$

and the exact velocity be $v(t)=\frac{t^{2}}{2}+0.01$. The model coefficients $r=\frac{1}{2}$ and $a(t)=0.01$, and the source term

$$
f(x, t)=1.98+\frac{t^{2-\alpha}}{\Gamma(3-\alpha)}+\frac{t^{1-\alpha}}{\Gamma(2-\alpha)}+2 t+t^{2} x+0.02 x,
$$

and we are to determine the average flow velocity $v(t)$ using the additional information $E(t)=1+(t+1)^{2}$. 


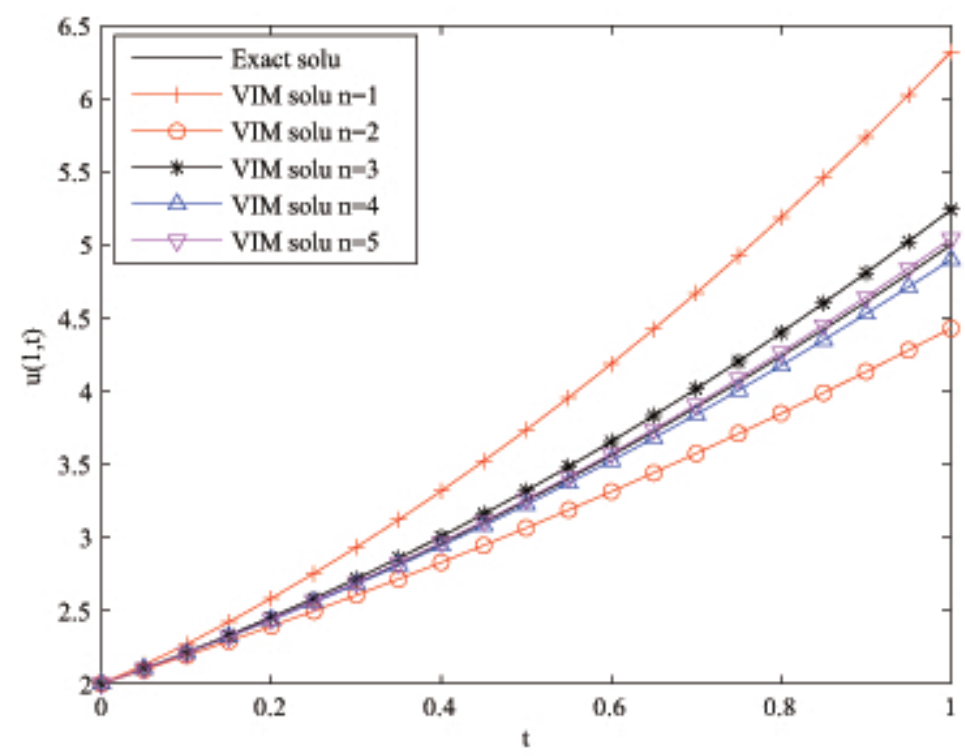

Figure 2. Exact and approximate solutions by VIM in Ex.4.2

Similarly as done in Ex. 4.1, we get a series of the approximated solutions by choosing the initial iteration $u_{0}=x^{2}+1$ given as follows:

$$
\begin{gathered}
u_{1}=x^{2}+(t+1)^{2}+\frac{t^{2-\alpha}}{\Gamma(3-\alpha)}+\frac{t^{3-\alpha}}{\Gamma(4-\alpha)} ; \\
u_{2}=x^{2}+(t+1)^{2}-\frac{t^{3-2 \alpha}}{2 \Gamma(4-2 \alpha)}-\frac{t^{4-2 \alpha}}{2 \Gamma(5-2 \alpha)} ; \\
\cdots \cdots ; \\
u_{n}=x^{2}+(t+1)^{2}+\frac{(-1)^{n-1} t^{n(1-\alpha)+1}}{2^{n-1} \Gamma(n(1-\alpha)+2)}+\frac{(-1)^{n-1} t^{n(1-\alpha)+2}}{2^{n-1} \Gamma(n(1-\alpha)+3)} .
\end{gathered}
$$

It is obvious that there holds

$$
u(x, t)=\lim _{n \rightarrow \infty} u_{n}(x, t)=x^{2}+(t+1)^{2} .
$$

Moreover, we have by (4.5) and (4.20)

$$
\begin{aligned}
v(t) & =\frac{f(1, t)-E^{\prime}(t)+a(t) u_{x}(1, t)-r \partial_{t}^{\alpha} E(t)}{u_{x}(1, t)} \\
& =\frac{f(1, t)-2(t+1)+0.01 \times 2-\frac{1}{2}\left[\frac{2 r^{2}-\alpha}{\Gamma(3-\alpha)}+\frac{2 t^{1-\alpha}}{\Gamma(2-\alpha)}\right]}{2} \\
& =\frac{t^{2}}{2}+0.01,
\end{aligned}
$$

which is just the exact flow velocity. As done in Ex.4.1, the approximate solutions of $u_{n}(x, t)(n=1, \cdots, 5)$ by VIM, and the exact solution $u(x, t)$ for $\alpha=0.8$ and $x=1$ are plotted in Fig.2, respectively.

It can be seen from Figures 1-2 that the approximate solutions by the VIM are convergent to the exact solutions only by several iteration steps, which demonstrates a fast convergence of the VIM algorithm.

\section{Conclusion}

We deal with determination problems of time-dependent coefficients in the time fractional diffusion equations using the variational iteration method. The variational iteration method is well-studied in giving semi-analytical approximate solutions for linear/nonlinear algebraic/differential equations, it is also effective for solving some nonlinear inverse timedependent coefficient problems in fractional differential equations. We will consider the convergence of the method and give more applications in other inverse problems.

\section{Acknowledgements}

This work is supported by the National Natural Science Foundation of China (no. 11871313), and the National Natural Science Foundation of Shandong Province, China (no. ZR2019MA021). 


\section{References}

Biazar, J., Gholamin, P., \& Hosseini, K. (2010). Variational iteration method for solving Fokker-Planck equation. J. Franklin Inst., 347, 1137-1146. https://doi.org/10.1016/j.jfranklin.2010.04.007

Chang, S.-H. (2016). Convergence of variational iteration method applied to two-point diffusion equation. Applied Mathematical Modelling, 40, 6805-6810. https://doi.org/10.1109/ICASI.2017.7988531

Chen, S., Liu, F., Jiang, X., Turner, I., \& Burrage, K. (2016). Fast finite difference approximation for identifying parameters in a two-dimensional space-fractional nonlocal model with variable diffusivity coefficients. SIAM J. Numer. Anal., 54, 606-624.

Cheng, J., Nakagawa, J., Yamamoto, M., \& Yamazaki, T. (2009). Uniqueness in an inverse problem for a one-dimensional fractional diffusion equation. Inverse Problems, 25, 115002. https://doi.org/10.1088/0266-5611/25/11/115002

Chi, G. S., Li, G. S., \& Jia, X. Z. (2011). Numerical inversions of source term in FADE with Dirichlet boundary condition by final observations. Computers and Mathematics with Applications, 62, 1619-1626. https://doi.org/10.1016/j.camwa.2011.02.029

Dehghan, M., \& Tatari, M. (2006). The use of He's variational iteration method for solving a Fokker-Planck equation. Phys. Scr., 74, 310-316. https://doi.org/10.1088/0031-8949/74/3/003

Fan, W., Liu, F., Jiang, X., \& Turner, I. (2018). Some novel numerical techniques for an inverse problem of the multiterm time fractinoal partial differential equation. Journal of Computational and Applied Mathematics, 336, 114-126. https://doi.org/10.1016/j.cam.2017.12.034

He, J. H. (1997). A new approach to nonlinear partial differential equations. Communications in Nonlinear Science and Numerical Simulation, 2, 230-235. https://doi.org/10.1016/S1007-5704(97)90007-1

He, J. H. (2000). Variational iteration method for autonomous ordinary differential systems. Applied Mathematics and Computation, 114, 115-123. https://doi.org/10.1016/S0096-3003(99)00104-6

He, J. H. (2007). Variational iteration methodłSome recent results and new interpretations. Journal of Computational and Applied Mathematics, 207, 3-17. https://doi.org/10.1016/j.cam.2006.07.009

Inc, M. (2008). The approximate and exact solutions of the space- and time-fractional Burgers equations with initial conditions by variational iteration method. J. Math. Anal. Appl., 345, 476-484. https://doi.org/10.1016/j.jmaa.2008.04.007

Jiang, W., \& Liu, N. (2017). A numerical method for solving the time variable fractional order mobile-immobile advection-dispersion model. Applied Numerical Mathematics, 119, 18-32.

Jin, B. T., \& Rundell, W. (2015). A tutorial on inverse problems for anomalous diffusion processes. Inverse Problems, 31, 035003. https://doi.org/10.1088/0266-5611/31/3/035003

Kian, Y., Oksanen, L., Soccorsi, E., \& Yamamoto, M. (2018). Global uniqueness in an inverse problem for time fractional diffusion equations. Journal of Differential Equations, 264, 1146-1170. https://doi.org/10.1016/j.jde.2017.09.032

Kilbas, A. A., Srivastava H. M., \& Trujillo, J. J. (2006). Theory and Applications of Fractional Differential Equations. Amsterdam: Elsevier. https://doi.org/10.3182/20060719-3-PT-4902.00008

Kumar, S., Kumar, A., \& Argyros, I. K. (2017). A new analysis for the Keller-Segel model of fractional order. Numerical Algorithm, 75, 213-218. https://doi.org/10.1007/s11075-016-0202-z

Li, G. S., Zhang, D. L., Jia X. Z., \& Yamamoto, M. (2013). Simultaneous inversion for the space-dependent diffusion coefficient and the fractional order in the time-fractional diffusion equation. Inverse Problems, 29, 065014. https://doi.org/10.1088/0266-5611/29/6/065014

Li, Z. Y., Imanuvilov, O. Y., \& Yamamoto, M. (2016). Uniqueness in inverse boundary value problems for fractional diffusion equations. Inverse Problems, 32, 015004. https://doi.org/10.1088/0266-5611/32/1/015004

Liu, F. W., Meerschaert, M. M., McGough, R. J., Zhuang, P. H., \& Liu, Q. X. (2013). Numerical methods for solvoing the multi-term time-fractinoal wave-diffusion. Fractional Calculus and Applied Analysis, 16, 9-25.

Liu, J. J., \& Yamamoto, M. (2010). A backward problem for the time-fractional diffusion equation. Applicable Analysis, 89, 1769-1788. https://doi.org/10.1080/00036810903479731

Liu, Q., Liu, F., Turner, I., Anh, V., \& Gu, Y. T. (2014). A RBF meshless approach for modeling a fracal mobile/immobile transport model. Applied Mathematics and Computation, 226, 336-347. https://doi.org/10.1016/j.amc.2013.10.008 
Liu, Y. K., Rundell, W., \& Yamamoto, M. (2016). Strong maximum principle for fractional diffusion equations and an application to an inverse source problem. Fractional Calculus and Applied Analysis, 19, 888-906. https://doi.org/10.1515/fca-2016-0048

Luchko, Y., Rundell, W., Yamamoto, M., \& Zuo, L. H. (2013). Uniqueness and reconstruction of an unknown semilinear term in a time-fractional reaction-diffusion equation. Inverse Problems, 29, 065019. https://doi.org/10.1016/S09694765(20)30014-X

Miller, L., \& Yamamoto, M. (2013). Coefficient inverse problem for a fractional diffusion equation. Inverse Problems, 29, 075013. https://doi.org/10.1088/0266-5611/29/7/075013

Nadeem, M., Li, F. Q., \& Ahmad, H. (2019). Modified Laplace variational iteration method for solving fourth-order parabolic partial differential equation with variable coefficients. Computers and Mathematics with Applications, 78, 2052-2062.

Podlubny, I. (1999). Fractional Differential Equations. San Diego: Academic.

Ruan, Z. S., Zhang, W., \& Wang, Z. W. (2018). Simultaneous inverison for the fractinal order and the space-dependent source term for the time-fractional diffusion equation. Applied Mathematics and Computation, 328, 365-379.

Sakamoto, K., \& Yamamoto, M. (2011). Initial value/boundary value problems for fractional diffusion-wave equations and applications to some inverse problems. Journal of Mathematical Analysis and Applications, 382, 426-447.

Sun, C. L., Li, G. S., \& Jia, X. Z. (2017). Simultaneous inversion for the diffusion and source coefficients in the multi-term TFDE. Inverse Problems in Science and Engineering, 25, 1618-1638. https://doi.org/10.1080/17415977.2016.1275612

Sun, L. L., Yan, B. X., \& Wei, T. (2019). Identification of time-dependent convection coefficient in a time-fractional diffusion equation. Journal of Computational and Applied Mathematics, 346, 505-517. https://doi.org/10.1016/j.cam.2018.07.029

Tapaswini, S., Chakraverty, S., \& Behera, D. (2015). Numerical solution of the imprecisely defined inverse heat conduction problem. Chinese Physics B, 24, 050203. https://doi.org/10.1088/1674-1056/24/5/050203

Wei, T., Li, X. L., \& Li, Y. S. (2016). An inverse time-dependent source problem for a time-fractional diffusion equation. Inverse problems, 32, 085003. https://doi.org/10.1088/0266-5611/32/8/085003

Wu, G. C. (2012). Variational iteration method for solving the time-fractional diffusion equations in porous medium. Chinese Physics B, 21, 120504. https://doi.org/10.1088/1674-1056/21/12/120504

Yamamoto, M., \& Zhang, Y. (2012). Conditional stability in determining a zeroth-order coefficient in a half-order fractional diffusion equation by a Carleman estimate. Inverse problems, 28, 105010. https://doi.org/10.1088/0266$5611 / 28 / 10 / 105010$

Yulita, M. R., Noorani, M. S. M., \& Hashim, I. (2009). Variational iteration method for fractional heat- and wave-like equations. Nonlinear Analysis: Real World Appl., 10, 1854-1869.

Zhang, H., Liu, F., Phanikumar, M. S., \& Meerschaert, M. M. (2013). A novel numerical method for the time variable fractional order mobile-immobile advection-dispersion model. Computers and Mathematics with Applications, 66, 693-701.

Zhang, Z. D. (2016). An undetermined coefficient problem for a fractional diffusion equation. Inverse Problems, 32, 015011. https://doi.org/10.1088/0266-5611/32/1/015011

Zhao, G. Z., Zhu, J., Yu, X. J., \& Xu, Y. (2010). Variational iteration method for solving compressible Euler equations. Chinese Physics B, 19, 070203.

\section{Copyrights}

Copyright for this article is retained by the author(s), with first publication rights granted to the journal.

This is an open-access article distributed under the terms and conditions of the Creative Commons Attribution license (http://creativecommons.org/licenses/by/4.0/). 